\title{
THE FUNDAMENTAL PRIME IDEALS OF A NOETHERIAN PRIME PI RING
}

\author{
by T. H. LENAGAN and EDWARD S. LETZTER
}

(Received 8th August 1988)

\begin{abstract}
Let $R$ be a noetherian prime PI ring and let $P$ be a prime ideal of $R$. There is a set of prime ideals, the fundamental prime ideals, associated with the injective hull of $R / P$ and denoted by $F u n d(P)$. The set $F$ und $(P)$ is finite, by a result of Müller. In this paper we give a natural description of $F$ und $(P)$ in terms of the trace ring of $R$ which strengthens Müller's result by establishing a uniform bound for the size of Fund $(P)$ for all primes $P$ in the ring.
\end{abstract}

1980 Mathematics subject classification (1985 Revision): 16A33, 16A38, 16A52, 16A64.

\section{Introduction}

Let $R$ be a prime noetherian PI ring, let $P$ be a prime ideal of $R$, and let $E$ denote the $R$-injective hull of $(R / P)_{R}$. Müller has shown that the set of fundamental prime ideals of $E$, Fund $(P)$, is finite. (If $P$ is maximal then the set of fundamental prime ideals is precisely the set of annihilators of irreducible subfactors of $E$.) In this note we show that Fund $(P)$ has a natural formulation in terms of the trace ring of $R$, and we present a "finite" procedure for determining Fund $(P)$. Müller's result follows from this description. In fact, we are able to prove the following: If $R$ is a prime noetherian PI ring then there exists a positive integer $n$ such that $|\mathrm{Fund}(Q)| \leqq n$ for all prime ideals $Q$ of $R$.

\section{The second layer condition}

Although our main interest here concerns prime ideals and injective modules for noetherian polynomial identity algebras, many of the basic ideas are valid in a wider context and we start by describing this context. (For unexplained terminology in what follows, consult $[5,8]$, or the specific references provided later-or at first reading assume that all rings have a polynomial identity and so are fully bounded.)

To begin, let $R$ be a noetherian ring, and let $P$ be a prime ideal of $R$. If $Q$ is also a prime ideal, and if there exists an $R-R$-bimodule factor of $Q \cap P / Q P$ which forms an $R / Q-R / P$-bimodule that is torsion free on each side, then there is said to be a right link (or direct link) from $Q$ to $P$, denoted by $Q \leadsto P$. A subset $X$ of $\operatorname{spec} R$ is said to be closed under right links if for each $Q \in X$ and each $Q^{\prime} \leadsto Q$ it follows that $Q^{\prime} \in X$. The right clique of $P$ is the smallest subset of $\operatorname{spec} R$ which contains $P$ and is closed under right links.

Next we consider injective modules over $R$. First, if $M$ is a right $R$-module, denote by 
$E(M)_{R}$ the $R$-injective envelope of $M$. Now let $U$ be a uniform right ideal of $R / P$. Observe that $E(R / P)_{R} \cong \bigoplus_{i=1}^{n} E(U)_{R}$, where $n$ is the Goldie rank of $R / P$. Hence in order to study $E(R / P)_{R}$ it is enough to study $E(U)_{R}$, an indecomposable injective module. Conversely, if $R$ is an FBN-ring then each indecomposable injective right module is isomorphic to $E(V)_{R}$ for some uniform right ideal $V$ of a prime factor of $R$, see [6].

Definition 1.1. Let $R$ be a noetherian ring. Then $R$ is said to satisfy the right second layer condition if for each prime ideal $P$ of $R$ the following property holds: If $M_{R} \hookrightarrow E(R / P)_{R}$ is a finitely generated $R$-module then there exists a series $0=M_{0} \subset \cdots \subset$ $M_{n}=M$ of right $R$-modules such that each $M_{i} / M_{i-1}$ is isomorphic to a right ideal of $R / P_{i}$ for some $P_{i}$ in the right clique of $P$. (In particular, $M P_{n} \ldots P_{1}=0$.) Further, $R$ is said to satisfy the second layer condition if it satisfies the right and left second layer conditions. Fully bounded noetherian rings, and in particular noetherian PI rings satisfy the second layer condition; see [5].

\section{Fundamental primes and ideal links}

Throughout this section let $R$ be a noetherian ring satisfying the second layer condition and let $P$ be a prime ideal of $R$. Choose a uniform module $U$ that is isomorphic to a right ideal of $R / P$ and set $E=E(U)_{R}$, the $R$-injective hull of $U$. We start by defining the fundamental series and fundamental prime ideals of $E$ and summarize the results that we need. Most of what we say is extracted from [4] and [5, Chapter 9].

Let $Y$ be a set of prime ideals of $R$. A semiprime ideal $S$ of $R$ is called $Y$-semiprime if each prime ideal minimal over $S$ is in $Y$. Define $X_{1}(P)=P$, and, for $n \geqq 1$,

$$
X_{n+1}(P)=\left\{Q \in \operatorname{spec}(R) \mid Q \leadsto Q_{n} \text { for some } Q_{n} \in X_{n}(P)\right\} \text {. }
$$

Thus $X=X(P)=\bigcup_{n=1}^{\infty} X_{n}(P)$ is the right clique of $P$. The set $X$ satisfies the incomparability condition: that is, if $Q_{1}, Q_{2} \in X$ and $Q_{1} \subseteq Q_{2}$ then $Q_{1}=Q_{2}$. The fundamental series $\left\{F_{n}\right\}$ of $E$ is defined as follows: $F_{1}=\operatorname{ann}_{E}(P)$, and for $n \geqq 1, F_{n+1}$ is the full inverse image in $E$ of the sum of annihilators in $E / F_{n}$ of all $X_{n+1}$-semiprime ideals of $R$. It is not difficult to see that $E=\bigcup_{n=1}^{\infty} F_{n}[4$, Lemma $5.4 ; 5,9.1 .2]$. We denote by $\operatorname{Fund}_{n+1}(P)$ the set of assassinator prime ideals of $E / F_{n}$ and set $\operatorname{Fund}(P)=\bigcup_{n=1}^{\infty} F_{\text {und }}(P)$, the fundamental prime ideals of $P$. Thus Fund $(P) \subseteq$ right clique $(P)$. However, in general Fund $(P) \neq \operatorname{right}$ clique $(P)$ : this is demonstrated in a spectacular manner by a result of Müller [10, Theorem 7] which states that if $R$ is a noetherian PI ring then Fund $(P)$ is finite, while even for a prime noetherian PI ring right clique $(P)$ may be infinite [9, p. 242].

Our original intention was to gain a better understanding of Müller's result in the prime case by finding some known finite set of prime ideals that would contain Fund $(P)$. In order to do this we develop a description of the fundamental prime ideals that is internal to the ring $R$. If $A \subseteq B$ are ideals of $R$, and if $Q, P$ are prime ideals of $R$ such that $B / A$ is naturally an $R / Q-R / P$-bimodule that is torsion free on both sides, then we say that there is an ideal link from $Q$ to $P$ via $B / A$ and write $Q \approx P$. More generally, if $R, S$ are prime noetherian rings and ${ }_{R} B_{S}$ is a bimodule that is torsion free 
and finitely generated on both sides then we say that there is a bond from $R$ to $S$. The following lemma gives some of the well-known properties concerning torsion and bonds.

Lemma 2.1. Let $R$ and $S$ be noetherian rings satisfying the second layer condition. Let $B$ be an $R$-S-bimodule that is finitely generated on each side.

(i) Suppose that $S$ is prime. If $B_{S}$ is a torsion $S$-module then $\operatorname{ann}(B)_{S} \neq 0$.

(ii) If $B$ is faithful on each side then the classical Krull dimensions of $R$ and $S$ are equal.

(iii) Suppose that $R$ and $S$ are prime rings and have the same classical Krull dimension. If $B_{S}$ is not a torsion module then some factor bimodule of $B$ forms $a$ bond from $R$ to $S$.

Proof. (i) This follows from [5, 5.1.2].

(ii) This is $[5,8.2 .8]$.

(iii) Suppose that $B_{S}$ is not torsion, let ${ }_{R} T_{S}$ be the right torsion bimodule, and let $\bar{B}=B / T$. Let ${ }_{R} \bar{A}_{S}$ be the left torsion bimodule of $\bar{B}$. Since $\bar{B}_{S}$ is torsion free $\bar{A}_{S}$ must be faithful, but since ${ }_{R} \bar{A}$ is torsion ${ }_{R} \bar{A}$ cannot be faithful. In view of (ii) and the assumption that the classical Krull dimensions of $R$ and $S$ coincide, we see that $\bar{A}=0$. Hence $\bar{B}$ is a bond from $R$ to $S$.

The next result is essentially in $[5,9.1 .2]$ or $[4$, Lemma 5.4] although in neither place is it precisely stated in this form.

Lemma 2.2. Let $R$ be a noetherian ring with the second layer condition and let $P$ be a prime ideal of $R$. Let $U$ be a uniform right ideal of $R / P$ and set $E=E(U)_{R}$. Then:

(i) If $M$ is a finitely generated submodule of $E$ with $M \subseteq F_{n}$ then there exist Fund $(P)$-semiprime ideals $S_{n}, \ldots, S_{2}$ of $R$ such that $M S_{n} \ldots S_{2} P=0$.

(ii) If $M$ is a finitely generated right submodule of $E$ then there exist prime ideals $P_{t}, \ldots, P_{1} \in$ Fund $(P)$ such that $M P_{t} \ldots P_{1}=0$.

Proof. (i) The proof is by induction on $n$. If $n=1$ then $M P=0$. If $n>1$ and $M$ is generated by $m_{1}, \ldots, m_{s}$ then there are Fund $(P)$-semiprime ideals $T_{1}, \ldots, T_{s}$ such that $m_{i} T_{i} \subseteq F_{n-1}$ and it follows that $M T \subseteq F_{n-1}$ where $T=T_{1} \cap \cdots \cap T_{s}$ is a Fund $(P)$ semiprime ideal of $R$. The result now follows by induction, since $M T$ is finitely generated.

(ii) This follows from (i) since each Fund $(P)$-semiprime ideal contains a product of prime ideals belonging to Fund $(P)$.

Theorem 2.3. Let $R$ be a noetherian ring satisfying the second layer condition and let $P$ be a prime ideal of $R$. Then $Q \in$ Fund $(P)$ if and only if $Q \approx P$.

Proof. Let $Q \in \operatorname{ass}\left(F_{n+1} / F_{n}\right)$ and choose $e \in F_{n+1} \backslash F_{n}$ such that $e Q \subseteq F_{n}$. There exist Fund $(P)$-semiprime ideals $S_{n}, \ldots, S_{2}$ such that $e Q S_{n} \ldots S_{2} P=0$ by Lemma 2.2(i). Set 
$B=Q S_{n} \ldots S_{2} \cap S_{n} \ldots S_{2} P$ and $A=Q S_{n} \ldots S_{2} P$ and note that $B / A$ is an $R / Q-R / P$. bimodule. If $B / A$ is torsion as an $R / P$-module then there exists an ideal $Y$ of $R$ with $P \varsubsetneqq Y$ such that $B Y \subseteq A$, by Lemma 2.1(i). In this case we see that $e B Y=0$, and it follows that $e B=0$, since $\operatorname{ann}_{E}(Y)=0$. Now $C=\left(Q \cap S_{n}\right)\left(S_{n} \cap S_{n-1}\right) \ldots\left(S_{2} \cap P\right)$ is a product of $n$ Fund $(P)$-semiprime ideals and $C \subseteq B$, so $e C=0$. However this implies that $e \in F_{n}$, by [4, Lemma 5.4]. Thus $B / A$ is not a torsion $R / P$-module and so, by Lemma 2.1(iii), $Q \approx P$.

Conversely, suppose that $Q \approx P$ via an ideal link $B / A$. Choose a right ideal $K$ of $R$ maximal such that $B \cap K=A$. Then there is an embedding of $B / A$ as an essential submodule of the right $R$-module $R / K$.

Since $B / A$ is finitely generated and torsion free as an $R / P$-module it embeds into a finite direct sum of copies of $E[5,2.2 .15]$, and hence $R / K$ also embeds in this way. Hence, by Lemma 2.2(ii), there are prime ideals $P_{1}, \ldots, P_{n} \in \mathrm{Fund}(P)$ such that $(R / K) P_{n} \ldots P_{1}=0$, and so $P_{n} \ldots P_{1} \subseteq K$. Thus $P_{n} \ldots P_{1} B \subseteq B \cap K=A$, so that $P_{n} \ldots P_{1} \subseteq Q$. Therefore, $P_{i} \subseteq Q$ for some $1 \leqq i \leqq n$. However $\operatorname{cl} . K \operatorname{dim}(R / Q)=\operatorname{cl} . K \operatorname{dim}(R / P)=$ cl. $K \operatorname{dim}\left(R / P_{i}\right)$, by Lemma $2.1\left(\right.$ ii), so $Q=P_{i}$ and $Q \in$ Fund $(P)$.

Although the following result may be well known, we give a proof since we have not been able to locate a specific reference.

Proposition 2.4. Let $R$ be a noetherian ring and let $x$ be a central element of $R$. If $P$ and $Q$ are prime ideals of $R$ such that $Q \approx P$ and $x \in P$ then $x \in Q$ and $\bar{Q} \approx \bar{P}$ in $\bar{R}=R / x R$.

Proof. Let $B / A$ be an ideal link from $Q$ to $P$. Since $x B=B x \subseteq B P \subseteq A$ it follows that $x \in Q$. Now $B \cap x^{m} R \subseteq B x R \subseteq A$, for some $m \geqq 1$, by the $A R$ property for $x R$, so $B \cap x^{m} R=A \cap x^{m} R$. Choose $n \geqq 0$ such that $B \cap x^{n} R \neq A \cap x^{n} R$, while $B \cap x^{n+1} R=$ $A \cap x^{n+1} R$. Note that the non-zero bimodule $\left(B \cap x^{n} R\right)+A / A$ gives an ideal link from $Q$ to $P$. Set $B^{\prime}=\left\{r \mid x^{n} r \in B\right\}$ and $A^{\prime}=\left\{r \mid x^{n} r \in A\right\}$. Then multiplication by the central element $x^{n}$ induces an $R-R$-bimodule isomorphism between $B^{\prime} / A^{\prime}$ and $\left(B \cap x^{n} R\right)+A / A$. Thus $B^{\prime} / A^{\prime}$ gives an ideal link from $Q$ to $P$. If $b^{\prime}=x r \in B^{\prime} \cap x R$ then $x^{n} b^{\prime}=x^{n+1} r \in B \cap x^{n+1} R=$ $A \cap x^{n+1} R \subseteq A$, so $b^{\prime} \in A^{\prime}$ and $B^{\prime} \cap x R \subseteq A^{\prime}$. Hence $B^{\prime}+x R / A^{\prime}+x R \cong B^{\prime} / A^{\prime}$ gives an ideal link from $Q$ to $P$ and the result follows.

An ideal $I$ of a noetherian ring $R$ is said to be polycentral if it can be generated by a sequence of elements $x_{1}, \ldots, x_{n}$ such that for each $i=1, \ldots, n$ the element $x_{i}$ is central modulo the ideal $\sum_{j=1}^{i-1} x_{j} R$. Obviously, a centrally generated ideal is polycentral.

Corollary 2.5. Let $R$ be a noetherian ring and let $I$ be a polycentral ideal of $R$. If $P$ and $Q$ are prime ideals of $R$ such that $Q \approx P$ and $I \subseteq P$ then $I \subseteq Q$ and $\bar{Q} \approx \bar{P}$ in $\bar{R}=R / I$.

\section{Lying over and contraction for ideal links}

Let $R G S$ be an embedding of rings. Let $Q$ be a prime ideal of $R$, and let $\tilde{Q}$ be a 
prime ideal of $S$. Following a standard convention, we say that $\tilde{Q}$ lies over $Q$ provided $Q$ is a minimal prime ideal over $\tilde{Q} \cap R$.

Theorem 3.1. Let $R \hookrightarrow S$ be an embedding of noetherian rings such that $S$ is a finitely generated right $R$-module. Suppose further that $R$ and $S$ satisfy the second layer condition. Let $P$ and $Q$ be prime ideals of $R$ such that $Q \approx P$. Then there exist prime ideals $\widetilde{Q}$ lying over $Q$ and $\widetilde{P}$ lying over $P$ such that $\tilde{Q} \approx \tilde{P}$.

Proof. The proof may be obtained by retracing the steps of the proofs of $[7,5.1(\mathrm{i})]$ and $[7,5.3]$ after first replacing the direct link $\left(Q_{\alpha} \cap Q_{\beta}\right) / A$ considered there by an arbitrary ideal link $C / A$. We provide an adaptation of this approach.

To begin, choose an ideal $K$ of $S$ maximal such that $K \cap R \subseteq P \cap Q$ and such that $Q / K \cap R \approx P / K \cap R$. To prove the proposition it suffices to show that the conclusion remains true for the embedding $R / K \cap R \hookrightarrow S / K$. Hence we may assume without loss of generality that $K=0$.

We next demonstrate a fundamental property of $S$. Let $C / A$ be an ideal link in $R$ from $Q$ to $P$, and let $I$ be a nonzero ideal of $S$. Suppose that $I \cap C \subseteq A$. Then $(I \cap R) \cdot C$ and $C \cdot(I \cap R)$ are both contained in $A$, which in turn implies that $I \cap R \subseteq P \cap Q$, and it is easy to see that $Q / I \cap R$ is ideal linked to $P / I \cap R$ via $(C+I \cap R) /(A+I \cap R)$. This statement contradicts our choice of $K$ above. We may therefore conclude that $I \cap C \nsubseteq A$.

We now show that $S$ is uniform as an $S$-S-bimodule. Let $C / A$ again be an ideal link from $Q$ to $P$, and let $I, J$ be ideals of $S$ such that $I \neq 0$ but $I \cap J=0$. By the previous paragraph, $I \cap C \nsubseteq A$. So let $C^{\prime}=I \cap C$, and let $A^{\prime}=I \cap A$. Then $C^{\prime} / A^{\prime}$ is an ideal link from $Q$ to $P$. However, $J \cap C^{\prime}=J \cap I \cap C=0 \subseteq A^{\prime}$. Hence the preceding paragraph shows that $J=0$. We conclude that $S$ is a uniform $S-S$-bimodule.

To describe the assassinators of $S$, first let $H \in$ ass $S_{S}$. Then there exists a nonzero ideal $I$ of $S$ such that $I H=0$. Let $C / A$ be the ideal link in $R$ from $Q$ to $P$. As shown above, $I \cap C \nsubseteq A$, and $I \cap C / I \cap A$ is an ideal link from $Q$ to $P$. Since $(I \cap C / I \cap A) \cdot(H \cap R)=0$, we see that $H \cap R \subseteq P$. A left-sided argument shows that if $G \in$ ass $S$, then $G \cap R \subseteq Q$. Further, since $S$ is a uniform $S$-S-bimodule, and since $S$ satisfies the second layer condition, it follows from $[5,8.3 .7]$ that ass $S_{S}$ consists of a single prime ideal $H$ and ass ${ }_{S} S$ consists of a single prime ideal $G$.

Now we describe the minimal prime ideals of $R$ and $S$. From the previous paragraph we let $\{G\}=$ ass ${ }_{S} S$. Now form a left affiliated series of $S$-R-bimodules for ${ }_{s} S_{R}$, say $0=S_{0} \subset \cdots \subset S_{n}=S$, and for each $i$ let $G_{i}=\operatorname{ann}_{S}\left(S_{i} / S_{i-1}\right)$. Observe that $G_{1}$ must be $G$. Next, form a right affiliated series of $S$-R-bimodules for ${ }_{s} S_{R}$, say $0=S_{0}^{\prime} \subset \cdots \subset S_{m}^{\prime}=S$, and for each $j$ let $T_{j}=\operatorname{ann}\left(S_{j}^{\prime} / S_{j-1}^{\prime}\right)_{R}$. Since ass ${ }_{S} S=\{G\}$, the $S-R$-sub-bimodule $M=$ $\{s \in S: G s=0\}$ is essential as a left $S$-submodule of ${ }_{S} S$. Hence ${ }_{s}\left(M \cap S_{1}^{\prime}\right)_{R}$ forms a bond from $S / G$ to $R / T_{1}$. Further, by $[5,8.3 .1]$, each $G_{i}$ is in the left clique of $G$, and each $T_{j}$ is in the right clique of $T_{1}$. Therefore, by repeated applications of Lemma 2.1(ii), we see that the factors $S / G_{1}, \ldots, S / G_{n}, R / T_{1}, \ldots, R / T_{m}$ all have the same classical Krull dimension. Noting that $G_{1} G_{2} \ldots G_{n}=T_{m} T_{m-1} \ldots T_{1}=0$, we see therefore that $\left\{G_{1}, \ldots, G_{n}\right\}$ is precisely the set of minimal prime ideals of $S$ and that $\left\{T_{1}, \ldots, T_{m}\right\}$ is precisely the set of minimal prime ideals of $R$. Further, again by Lemma 2.1(ii), it must be the case that the minimal prime ideals of $R$ and $S$ are closed with respect to ideal links. 
Next we prove that $P$ and $Q$ are minimal prime ideals of $R$ : Let $B_{R}$ be an $R$-submodule of $S_{R}$ maximal such that $B \cap C=A$, where $C / A$ is again an ideal link from $Q$ to $P$. So $C / A$ may be considered as an essential right $R$-submodule of $(S / B)_{R}$. Therefore, by $[5,7.1 .2], S P_{1} \ldots P_{t} \subseteq B$ for some prime ideals $P_{1}, \ldots, P_{t}$ in the right clique of $P$. The choice of $B$ now guarantees that $S P_{1} \ldots P_{t} \cap C \subseteq A$, and the original choice of $K$ above thus implies that there exists no nonzero ideal $L$ of $S$ such that $L \subseteq S P_{1} \ldots P_{t}$. In other words, $S / S P_{1} \ldots P_{t}$ is faithful as a left $S$-module. Since $S / S P_{1} \ldots P_{t}$ is a right $\left(R / P_{1} \ldots P_{t}\right)$-module, it follows from Lemma 2.1 (ii) that $\operatorname{cl} K \operatorname{dim}\left(R / P_{1} \ldots P_{t}\right) \geqq$ cl. $K \operatorname{dim}(S)=$ cl. $K \operatorname{dim}(R)$. Hence the classical Krull dimension of $R / P_{1} \ldots P_{t}$ is equal to the classical Krull dimension of $R$, and it follows that some $P_{i}$ is a minimal prime ideal. We saw earlier that the set of minimal prime ideals of $R$ was closed under ideal links. Hence the set $\left\{P_{1}, \ldots, P_{t}, Q, P\right\}$ consists entirely of minimal prime ideals. In particular, $P$ and $Q$ are minimal.

To finish, let $\{G\}=$ ass $S$ and $\{H\}=$ ass $S_{S}$ as above. We have seen that $G \cap R \subseteq Q$ and $H \cap R \subseteq P$. Since $P$ and $Q$ are minimal we see then that $G$ lies over $P$ and that $H$ lies over $Q$. Since $s_{s}$ is a uniform bimodule, $\{s \in S \mid G s=0\} \cap\{s \in S \mid s H=0\} \neq 0$, and this ideal provides an ideal link in $S$ from $G$ to $H$. This fact completes the proof.

Recall that a ring embedding $R \hookrightarrow S$ is called a finite centralizing extension provided $S=R c_{1}+\cdots+R c_{n}$ for some elements $c_{1}, \ldots, c_{n} \in S$ such that $r c_{i}=c_{i} r$ for all $r \in R$ and each $1 \leqq i \leqq n$. Also recall that if $R \hookrightarrow S$ is a finite centralizing extension, and if $P \in \operatorname{spec} S$, then $P \cap R \in \operatorname{spec} R$. See $[8,10.2]$ for details.

Theorem 3.2. Let $R \hookrightarrow S$ be a finite centralizing extension of noetherian rings. If $P$ and $Q$ are prime ideals of $S$ such that $Q \approx P$, then $Q \cap R \approx P \cap R$.

Proof. Let $S=R c_{1}+\cdots+R c_{n}$, where each $c_{i}$ centralizes $R$. For each $1 \leqq i \leqq n$, let $S_{i}=R c_{1}+\cdots+R c_{i}$, and let $S_{0}=0$. Observe that each $S_{i}$ is an $R-R$-sub-bimodule of ${ }_{R} S_{R}$. We may define, for each $i$, an $R$-R-bimodule homomorphism $\theta_{i}: R \rightarrow S_{i} / S_{i-1}$ via $r \rightarrow r c_{i}+S_{i-1}$. It is straightforward to check that $\theta_{i}$ is an $R-R$-bimodule homomorphism, given that $c_{i}$ centralizes $R$. Moreover, if $X_{i}=\operatorname{ker} \theta_{i}$, then $X_{i}$ is a two-sided ideal of $R$ and $R / X_{i}$ is isomorphic to $S_{i} / S_{i-1}$ as an $R-R$-bimodule.

Now let $P$ and $Q$ be prime ideals of $S$ such that $C / A$ is an ideal link in $S$ from $Q$ to $P$. Consider the two $R-R$-bimodule series $0=S_{0} \subset S_{1} \subset \cdots \subset S_{n}=S$ and $0 \subset A \subset C \subset S$. By the Schreier Refinement Theorem, there exist an $R-R$-sub-bimodule $B$ of $S$ with $A \varsubsetneqq B \subset C$, and an $R-R$-bimodule subfactor $W$ of $S_{i} / S_{i-1}$, for some $i$, such that $W$ is isomorphic to $B / A$ as an $R-R$-bimodule.

We claim that $B / A$, and hence $W$, is a bond from $R / Q \cap R$ to $R / P \cap R$. If $B / A$ is not a bond then, without loss of generality, suppose that $B / A$ has a nonzero sub-bimodule $B^{\prime} / A$ that is torsion as a right $R / P \cap R$-module. Then there exists an ideal $X$ of $R$ strictly bigger than $P \cap R$ such that $B^{\prime} X \subseteq A$. But then $S X S \subseteq \operatorname{ann}\left(B^{\prime} / A\right)_{S}$, since $S$ is a centralizing extension of $R$; and so $S X S \subseteq P$, thus contradicting $X \nsubseteq P \cap R$. Now the map $\theta_{i}$ above shows that $W$ is isomorphic to an $R-R$-bimodule subfactor $V$ of $R / X_{i}$. Since $V$ will equal $K / L$ for some pair of ideals $K, L$ of $R$, it follows that $Q \cap R \approx P \cap R$. 
Remark. In [3, Proposition 5] it is shown that if $P, Q \in \operatorname{spec} S$ and $Q \leadsto P$ then either $Q \cap R \leadsto P \cap R$ or $Q \cap R=P \cap R$, for $R \hookrightarrow S$ a finite centralizing extension.

\section{Rings satisfying a polynomial identity}

In this section we apply the results of the previous two sections to the study of Fund $(P)$ for a prime ideal $P$ of a noetherian prime PI ring $R$. The main facts that we need concerning PI rings can be found in [8, Chapter 13, especially $\$ 9$ ]. Recall that a prime PI ring $R$ is contained in a possibly larger subring of its quotient ring, the trace ring of $R$, written $T=T(R)$. If $R$ is noetherian then $T$ is a finite centralizing extension of $R$. Noetherian PI rings satisfy the second layer condition so our earlier results are all available for use in this setting. Our first aim is to transfer to $T$ the problem of finding ideal links in $R$. The advantage of working in the trace ring is that $T$ is an integral extension of its centre $Z$, and, as we shall see in Proposition 4.1, there are only finitely many prime ideals of $T$ with a given contraction to $Z$.

We start by introducing two more concepts of linkage between prime ideals of $R$. Let $P$ and $Q$ be prime ideals of $R$. Following [3] we say that $P$ and $Q$ are trace-linked if there are prime ideals $\widetilde{P}$ and $\tilde{Q}$ of $T$ such that $\widetilde{P} \cap R=P, \widetilde{Q} \cap R=Q$, and $\tilde{P} \cap Z=\widetilde{Q} \cap Z$. The reader should be warned that this is not an equivalence relation on $\operatorname{spec}(R)$. Denote by $\operatorname{Tr}(P)$ the set of prime ideals that are trace-linked to $P$.

Proposition 4.1. Let $R$ be a noetherian prime PI ring with PI-degree $n$ and suppose that the trace ring $T$ of $R$ can be generated as an $R$-module by $m$ elements that centralize $R$. Then for any prime ideal $P$ or $R$,

$$
|\operatorname{Tr}(P)| \leqq m n
$$

Proof. Let $P_{1}, \ldots, P_{r}$ be the distinct prime ideals of $T$ such that $P_{i} \cap R=P$. By $[11$, Theorem 3.4], $r \leqq m$. For any fixed $i$, the maximal number of prime ideals $Q$ of $T$ such that $P_{i} \cap Z=Q \cap Z$ is $n$. This follows from [1, Proposition 5] once one has reduced to the case that $P_{i} \cap Z$ is a maximal ideal of $Z$, by using [2, Lemma 2]. The prime ideals of $R$ given by $Q \cap R$ for such primes $Q$ form $\operatorname{Tr}(P)$, so $|\operatorname{Tr}(P)| \leqq m n$.

Let $P$ and $Q$ be prime ideals of the noetherian prime PI ring $R$. We say that there is a trace-ideal-link from $Q$ to $P$, written $Q \approx{ }_{T} P$, if there exist prime ideals $\tilde{P}$ and $\tilde{Q}$ of $T$ such that $\tilde{P} \cap R=P, \tilde{Q} \cap R=Q$, and $\tilde{Q} \approx \tilde{P}$.

Lemma 4.2. (i) $Q \approx P$ if and only if $Q \approx_{T} P$.

(ii) If $Q \approx_{T} P$ then $Q \in \operatorname{Tr}(P)$.

Proof. (i) This is immediate from Theorems 3.1 and 3.2 .

(ii) Let $\widetilde{P}$ and $\tilde{Q}$ be prime ideals of $T$ such that $\tilde{P} \cap R=P, \widetilde{Q} \cap R=Q$, and $\widetilde{Q} \approx \tilde{P}$. Then $\tilde{Q} \cap Z=\tilde{P} \cap Z$, since multiplication by central elements passes across the ideal link, and so $Q$ and $P$ are trace-linked.

Remark. K. A. Brown has suggested the following alternative proof that $Q \approx_{T} P$ 
implies $Q \approx P$ : Let $Q, P \in \operatorname{spec} R$ and let $\widetilde{Q}, \tilde{P}$ be prime ideals of $T$ such that $\tilde{Q} \cap R=Q$ and $\tilde{P} \cap R=P$. Suppose that $\tilde{B} / \tilde{A}$ is an ideal link in $T$ from $\tilde{Q}$ to $\tilde{P}$. There exists a nonzero ideal $I$ of $T$ such that $I \subseteq R$, and $I$ must contain a regular central element $c$. (See $[8,13.9 .6]$ and $[8,13.6 .4]$.) Hence $\tilde{B} c / \tilde{A} c$ is an ideal link in $R$ from $Q$ to $P$.

Theorem 4.3. If $R$ is a noetherian prime $P I$ ring and $P$ is a prime ideal of $R$ then Fund $(P) \subseteq \operatorname{Tr}(P)$.

Proof. Let $Q \in$ Fund $(P)$. There is an ideal link $Q \approx P$, by Theorem 2.3. Thus the result follows, by Lemma 4.2 .

Corollary 4.4. Let $R$ be a noetherian prime PI ring with trace ring $T$. Suppose that $T$ can be generated as an $R$-module by $m$ elements that centralize $R$, and suppose that the $P I-$ degree of $R$ is $n$. If $P$ is any prime ideal of $R$ then $\mid$ Fund $(P) \mid \leqq m n$.

Proof. This follows immediately from Theorem 4.3 and Proposition 4.1.

Of course, Theorem 4.3 suggests the obvious question as to whether $\operatorname{Fund}(P)=\operatorname{Tr}(P)$. We have not yet been able to answer the following related question: Let $R$ be a noetherian prime ring that is finitely generated as a module over its centre $A$. Suppose that $A$ is a local ring with maximal ideal $M$. If $P$ and $Q$ are maximal ideals of $R$ we know that $P \cap A=M=Q \cap A$. Is there an ideal link from $Q$ to $P$ ?

Leaving aside this problem, we finish the section by outlining a "finite" procedure for finding $\operatorname{Fund}(P)$ when $P$ is a prime ideal of the noetherian prime PI ring $R$. By Lemma 4.2 and Theorem 2.3 we need only find the prime ideals of $R$ that are trace-ideal-linked to $P$. Thus if $\widetilde{P}_{1}, \ldots, \widetilde{P}_{r}$ are the prime ideals of $T$ such that $\tilde{P}_{i} \cap R=P$, then what is left to determine is which prime ideals of $T$ are ideal linked to some $\tilde{P}_{i}$. This transfers the problem of determining Fund $(P)$ to the problem of finding those prime ideals of $T$ which are ideal linked to at least one $\widetilde{P}$ in $\left\{\tilde{P}_{1}, \ldots, \tilde{P}_{r}\right\}$.

Now fix such a $\tilde{P} \in\left\{\tilde{P}_{1}, \ldots, \tilde{P}_{r}\right\}$. Recall for a prime ideal $\tilde{Q}$ of $T$ that if $\tilde{Q} \approx \tilde{P}$ then $\tilde{Q} \cap Z=\tilde{P} \cap Z$. Let $M=\tilde{P} \cap Z$. Hence to find those prime ideals of $T$ which are ideal linked to $\tilde{P}$, we need only consider the (finite) set of prime ideals of $T$ which contract to $M$. Further, if $\mathscr{C}=A \backslash M$, then it is straightforward to check that $\tilde{Q} \approx \tilde{P}$ in $T$ if and only if $\widetilde{Q} \mathscr{C}^{-1} \approx \widetilde{P} \mathscr{C}^{-1}$ in the localisation $T \mathscr{C}^{-1}$. Next, by Corollary 2.5 , if we let $A=$ $T \mathscr{C}^{-1} / T M \mathscr{C}^{-1}$, if we let $\theta$ be the natural map from $T$ to $A$, then $\tilde{Q} \approx \tilde{P}$ in $T$ if and only if $\theta(\tilde{Q}) \approx \theta(\widetilde{P})$ in $A$. Observe that $A$ is artinian, and observe that we have reduced the problem of finding prime ideals $\tilde{Q}$ linked to $\tilde{P}$ to the problem of locating ideal links in $A$. Finally, note that the ideal links in $A$ will be completely determined by an $A-A$ bimodule composition series for $A$; see [5, pp. 142-144].

Acknowledgements. This work was done while the second author was visiting the University of Edinburgh, partly funded by the Centenary Fund of Edinburgh Mathematical Society and the National Science Foundation of America. He thanks these institutions for their hospitality and financial support. 


\section{REFERENCES}

1. A. Braun, An additivity principle for PI rings, J. Algebra 96 (1985), 433-441.

2. A. Braun and L. W. Small, Localization in prime noetherian PI rings, Math. Z. 193 (1986), 323-330.

3. A Braun and R. B. Warfield, JR., Symmetry and localization in noetherian prime PI rings, $J$. Algebra, to appear.

4. K. A. Brown and R. B. Warfield, JR., The influence of ideal structure on representation theory, J. Algebra 116 (1988), 294-315.

5. A. V. Jategaonkar, Localization in Noetherian Rings (London Math. Soc. Lecture Note, Vol. 98, Cambridge Univ. Press, Cambridge, 1986).

6. G. R. Krause, On fully left bounded left noetherian rings, J. Algebra 23 (1972), 88-99.

7. E. S. LetzTER, Prime ideals in finite extensions of noetherian rings, J. Algebra, to appear.

8. J. C. McConnell and J. C. Robson. Noncommutative Noetherian Rings (Wiley, New York, 1988).

9. B. J. Múller, Localization in fully bounded noetherian rings, Pacific J. Math. 67 (1976), 233-245.

10. B. J. Múller, Two-sided localization in noetherian PI rings, J. Algebra 63 (1980), 359-373.

11. J. C. Robson, Prime ideals in ntermediate extensions, Proc. London Math. Soc. (3) 44 (1982), 372-384.

Mathematics Department

King's BUILDINGS

MAYFIELD RoAD

EDINBURGH EH9 3JZ
Mathematics Department

UNIVERSITY OF WASHINGTON, GN-50

Seattle

WASHINGTON 98195 